\title{
Self-Perceived Preparedness to Work Independently Among Graduating Dental Students in Saudi Arabia: Traditional Versus Integrated Curriculum
}

\author{
Sudhakar Vundavalli1 (iD
}

${ }^{1}$ Department of Preventive Dentistry, College of Dentistry, Jouf University, Al Jouf, King of Saudi Arabia.

Author to whom correspondence should be addressed: Sudhakar Vundavalli, Department of Preventive Dentistry, College of Dentistry, Sakaka, Al Jouf, King of Saudi Arabia. Phone: +966 533982791. E-mail: dr.sudhakar.vundavalli@jodent.org.

Academic Editors: Alessandro Leite Cavalcanti and Wilton Wilney Nascimento Padilha

Received: 15 December 2019 / Accepted: 27 March 2020 / Published: 13 April 2020

How to cite this article: Vundavalli S. Self-perceived preparedness to work independently among graduating dental students in Saudi Arabia: traditional versus integrated curriculum. Pesqui Bras Odontopediatria Clín Integr. 2020; 20:e5460. https://doi.org/10.1590/pboci.2020.066

\begin{abstract}
Objective: To investigate the perceived preparedness of graduating dental students to work independently and to compare these perceptions between Traditional and Integrated curriculums of dentistry in Saudi Arabia. Material and Methods: A pretested self-administered questionnaire was filled by 258 dental students from four dental institutions across the country. The questionnaire covered 10 domains consisting of sixty suitable questions. Students were asked to mark their responses at one of five levels of performance for each of the selected skills ranging from 0 (not yet learned) to 4 (can do it confidently). Mean scores for each subscale of the Questionnaire were calculated by individual items and for all comparison, $\mathrm{p} \leq 0.05$ was considered statistically significant. Results: 253 graduating students were included in the final analysis, out of which 108 belongs to the Integrated curriculum group and 145 from Traditional curriculum. Overall scores of Traditional curriculum students $(176 \pm 4.8)$ were more compared to Integrated curriculum students $(161 \pm 3.2)$, which are statistically significant. Integrated curriculum students had more selfperceived skill scores in Orthodontics, Conservative/Operative Dentistry \& Endodontics, Dental Public Health and Drug \& Emergency management group of clinical skills. Overall, perceived preparedness to practice dentistry for all participants is $60.5 \%$ and traditional curriculum students expressed more preparedness (68\%) than Integrated curriculum (53\%). Conclusion: Overall, preparedness to practice independently is unsatisfactory compared to other countries. The Ministry of Education (KSA) should take necessary actions to bridge the gap between training dentists and to be competent practitioners.
\end{abstract}

Keywords: Dental Curriculum; Dental Education; Dental Graduate; Self-Assessment. 


\section{Introduction}

Saudi Arabia (KSA) is the largest country in the Middle East, with 34.2 million population [1]. However, the Country's healthcare system consists of both government and private health care providers, but the public health care system caters for the majority of the population and it holds good for Health care manpower training [2]. The first formal dental institution in Saudi Arabia was established at King Saud University, Riyadh, in the year 1976 and currently, more than twenty dental institutions offering graduation course in Dentistry [2].

The present dental education system in KSA has some serious issues that will need to be addressed to be able to produce efficient manpower in dentistry to match the burgeoning dental needs of the people [3]. Dental students in KSA are trained to excel in an institutional setup, but there appears to be a disengage between what knowledge and skills acquired during their graduation and what is required for clinical practice settings [3]. In an independent clinical setup, it is mandatory to have clinical problem-solving skills and practical knowledge regarding a variety of patients/dental problems are necessary.

Dental education and curriculum development in various countries has been regulated by centralized bodies such as the Commission for Dental Accreditation in the USA [4] and the Dental Council of India for India [5]. However, is Saudi Arabia, no single formal agency/body regulates dental education, so different dental schools follow different curriculums [6]. The role of National Commission for Academic Accreditation and Assessment [NCAAA] has been limited only to assess the quality of delivering the prescribed curriculums/programs and does not involve in assessing the appropriateness of curriculum's. The traditional semester based curriculums was followed in the majority of the dental institutions and integrated curriculum was recently introduced in few institutions [7]. A study done in Hong Kong to evaluate the perceived prepared of graduating students among these curriculums reported that there is no significant difference in graduates' perception about their self-perceived preparedness to dental practice [8].

Competency statements define the knowledge, clinical skills, attitude, and values that a dental graduate must possess to be able to work independently in real community settings [9]. Very little information is available about students' own perceptions of their competencies and professional preparation based on their undergraduate program, especially in comparison of the perceptions of students from Integrated curriculum and Traditional curriculum. Moreover, a disconnection between educational institutions and communities has been denounced in the literature analysing dental teaching in Saudi Arabian universities [3].

There was a study conducted on third-year graduate students to compare Traditional dental curriculum with Integrated curriculum and it was reported that Integrated curriculum helped in improving clinical problem-solving capabilities and students were more confident about their skills than the traditional curriculum students [10]. One of the major limitations of this study was the collection of information from third academic year students because the third year is too early to judge the curriculum, as real clinical training starts in the fourth academic year till sixth year (internship) in both curriculums.

No studies have been reported from Saudi Arabia regarding the comparison between two dental curriculums in graduating students. Therefore, it should be interesting to explore the perceptions of dental interns who are about to graduate about their own competencies and to compare the perceptions with those who underwent training in different curriculums. This study tested the hypothesis that there is no difference in the perceptions of dental students about their own competencies between Traditional and Integrated dental curriculums. 


\section{Material and Methods}

Study Design

This is a cross-sectional study among dental interns who are about to complete their graduation from four dental institutions. Development of the questionnaire, validation, data entry and statistical analysis was done as per study protocol. Data was collected based on the same methods and questionnaire as per study protocol.

\section{Study Participants}

This study included graduating dental students who are in doing their internship from fur dental institutions. An initial permission letter was sent to the Dean/Head of the institution of each of the selected dental institution and this letter discussed the background and goals of the research as well as requirements for participation and timetables for completion. All the interns in the four dental institutions were invited to participate in the study. They were requested for a classroom meeting to provide information about this research and procedures to complete the questionnaire. Those who agreed to participate (258 of the 278) gave informed consent to complete the questionnaire and no further sampling was done among the people who gave consent. The participants represented about $90 \%$ of the students who are about to graduate from the selected four dental institutions in the year 2020.

\section{Data Collection Tool}

The questionnaire was designed from competency statements mentioned from various institutions in Canada [9] and the American Association of Dental Schools (AADS) competencies for the new graduate dentist [11]. The questionnaire included 10 domains (Table 1) under which sixty questions were included: General patient management (seven questions), Practice Management (seven questions), Periodontology (four questions), Conservative/Operative Dentistry \& Endodontics (seven questions), Oral Rehabilitation/Prosthodontics (five questions), Orthodontics (four questions), Managing Paediatric patients and people with Special Needs (six questions), Oral and Dento-facial Surgery (nine questions), Dental Public Health (six questions), and Drug and Emergency Management (five questions) and at the end single dichotomous question was asked about the overall perceived preparedness to practice dentistry independently.

A panel of three experienced practicing dentists and two academicians reviewed the questionnaire and the objectives of the study. They were asked to give their opinions separately, based on their clinical and teaching experience, on the significance of each question to the required competencies and study objectives in the context of two current dental curriculums. They also provided suggested corrections/modifications and some changes were done as per their feedback. The questionnaire was then pretested on ten interns representing the study population. They were enquired about the questionnaire's clarity, it's comprehensiveness, appropriateness of instructions, and the time required to fill and their feedback steered to some minor modifications. The final questionnaire was tested for internal consistency with Cronbach's alpha (0.88).

Table 1. Dental competency domains and number of questions.

\begin{tabular}{clc}
\hline \multicolumn{1}{c}{ Clinical Skills } & No. of Items \\
\hline 1 & General Patient Management & 7 \\
2 & Practice Management & 7 \\
3 & Periodontology & 4
\end{tabular}




\begin{tabular}{clc}
4 & Conservative/Operative Dentistry \& Endodontics & 7 \\
5 & Oral Rehabilitation/Prosthodontics & 5 \\
6 & Orthodontics & 4 \\
7 & Managing Paediatric Patients and Children with Special Needs & 6 \\
8 & Oral and Dento-Facial Surgery & 9 \\
9 & Dental Public Health & 6 \\
10 & Drug and Emergency Management & 5 \\
\hline
\end{tabular}

\section{Data Collection}

Researchers collected the data directly from participants of each school. All the graduating students present on the day of data collection were invited for a classroom meeting in which the objectives of the study were explained, encouraged to participate voluntarily and the students were requested to fill the questionnaire under the supervision of the researchers. Filled questionnaires were checked for completeness. In the questionnaires, the participants were asked to rate their self-perceived competencies at one of five levels of performance for each of the selected skills ranging from 0 (not yet learned) to 4 (can do it confidently).

\section{Data Analysis}

Mean score of each of the questionnaire were calculated by averaging the scores for individual questions. The Student's t test was used to compare the means between two groups and Chi-square test for nominal data. Data was analysed using Statistical Software for Social Sciences (SPSS) version 20 (IBM SPSS., Chicago, IL, USA).

Ethical Aspects

The study was approved by the Local Committee for Bioethics, Jouf University, Protocol No. 07$03 / 41$.

\section{Results}

Out of 258 participants, five questionnaires were excluded as they were incomplete and uninformative and the final analysis included 253 participants, out of which 145 were from Traditional curriculum and 108 were from Integrated Curriculum. Overall scores of Traditional curriculum students (176 \pm 4.8$)$ were more compared to Integrated curriculum students $(161 \pm 3.2)$, which is statistically significant (Table 2). Integrated curriculum students had more self-perceived skill scores in Orthodontics, Conservative/Operative Dentistry \& Endodontics, Dental Public Health and Drug \& Emergency Management group of clinical skills. Traditional curriculum students had high self-perceived skills in general patient management, prosthodontics and oral \& maxillofacial surgery groups of skills than Integrated students (Table 2).

Table 2. Student's self-perceived clinical skills according to type of curriculum.

\begin{tabular}{|c|c|c|c|c|}
\hline \multirow[b]{2}{*}{ Clinical Skills } & \multirow{2}{*}{$\begin{array}{c}\text { Total } \\
\text { Items Score }\end{array}$} & \multicolumn{2}{|c|}{ Curriculum } & \multirow[b]{2}{*}{ p-value } \\
\hline & & $\begin{array}{l}\text { Traditional } \\
\text { Mean (SD) }\end{array}$ & $\begin{array}{l}\text { Integrated } \\
\text { Mean (SD) }\end{array}$ & \\
\hline General Patient Management & 28 & $19 \pm 1.2$ & $17 \pm 0.8$ & 0.062 \\
\hline Practice Management & 28 & $16 \pm 0.4$ & $14 \pm 0.5$ & 0.162 \\
\hline Periodontology & 16 & $14 \pm 0.3$ & $12 \pm 0.2$ & 0.262 \\
\hline Conservative/Operative Dentistry & 28 & $16 \pm 1.1$ & $22 \pm 2.8$ & $<0.001$ \\
\hline
\end{tabular}




$\begin{array}{lcccc}\text { Oral Rehabilitation/Prosthodontics } & 20 & 14 \pm 0.8 & 11 \pm 1.3 & 0.021 \\ \text { Orthodontics } & 16 & 9 \pm 0.4 & 13 \pm 1.2 & <0.001 \\ \text { Managing Children and Patients with Special Needs } & 24 & 12 \pm 1.8 & 16 \pm 2.2 & 0.014 \\ \text { Oral and Maxillofacial Surgery } & 36 & 26 \pm 1.1 & 20 \pm 1.2 & 0.304 \\ \text { Dental Public Health } & 24 & 11 \pm 1.6 & 16 \pm 1.8 & 0.003 \\ \text { Drug and Emergency Management } & 20 & 10 \pm 1.5 & 15 \pm 1.3 & 0.238 \\ \quad \text { Overall } & 240 & 176 \pm 4.8 & 161 \pm 3.2 & 0.046\end{array}$

All questions in each of the 10-domains were analysed to assess an overall view of the student's perception about their competency levels. Table 3 depicts the top five items and bottom five items that were rated by students as their highest and lowest levels of competencies. Collecting chair-side information from the patient and performing amalgam restorations were top two items rated by all students (100\%). Performing simple extractions (97\%), Knowledge about dental ethics (96.6\%) and administering local anaesthetics (96.3\%) were the next three items perceived by students in top five.

Table 3. Self perceived rating for skills about their own competencies: the top five and bottom five categories.

\begin{tabular}{ccc}
\multicolumn{1}{c}{ Competency Item } & Domain & Response* $^{*}$ \\
\hline Top Five & 1 & $100.0 \%$ \\
A] Record and interpret patients medical, social, and dental history & 4 & $100.0 \%$ \\
B] Restore teeth with amalgam restorations & 8 & $97.0 \%$ \\
C] Perform simple extractions & 9 & $96.6 \%$ \\
D] Knowledge about dental ethics & 10 & $96.3 \%$ \\
E] Administer local anaesthetics & & $18.0 \%$ \\
Bottom Five & 3 & $21.2 \%$ \\
A] Apply evidence-based dentistry & 4 & $24.5 \%$ \\
B] Perform periodontal surgery for crown lengthening & 6 & $25.0 \%$ \\
C] Perform multi-root canal treatment & 7 & $24.8 \%$ \\
D] Perform orthodontic treatment planning & 3 \\
E] Manage medically compromised patients & & \\
\hline
\end{tabular}

*Proportion of respondents who can do the task confidently.

Knowledge in applying evidence-based dentistry (18\%) was the ranked at bottom among the skills that students perceived of least competence, followed by performing periodontal surgery for crown lengthening (21.2\%), Performing multi-root canal treatment (24.5\%), Orthodontic treatment planning (25\%) and ability to manage medically compromised patients $(24.8 \%)$.

Students overall perceived preparedness to practice dentistry independently is $60.5 \%$ and Traditional curriculum cohorts expressed more preparedness than Integrated curriculum cohorts (Table 4).

Table 4. Perceived preparedness to practice dentistry independently.

\begin{tabular}{|c|c|c|c|c|c|c|c|}
\hline \multirow[t]{2}{*}{ Perception } & \multicolumn{2}{|c|}{ Traditional Curriculum } & \multicolumn{2}{|c|}{ Integrated Curriculum } & \multicolumn{2}{|c|}{ Overall* } & \multirow[t]{2}{*}{ p-value* } \\
\hline & $\mathrm{N}$ & $\%$ & $\mathrm{~N}$ & $\%$ & $\mathrm{~N}$ & $\%$ & \\
\hline Yes & 99 & 68.0 & 57 & 53.0 & 156 & 60.5 & $<0.05$ \\
\hline No & 46 & 32.0 & 51 & 47.0 & 97 & 39.5 & \\
\hline Total & 145 & 57.3 & 108 & 42.7 & 253 & 100.0 & \\
\hline
\end{tabular}

*Chi-square test.

\section{Discussion}


This study is the first of this type of survey comparing self-perceived competencies among dental students from two different curriculums from Saudi Arabia. Competencies embody minimum expected standards of any fresh graduate who deals with patients in real independent clinical settings [9]. Other than the routine assessments of students as a part of the academic program, assessing the students' competence in handling dental patients in independent clinical practice settings is considered a litmus test to evaluate the efficiency of any dental Curriculum [12]. In this study, the students perceived to be less confident about their ability to practice dentistry independently; nearly $40 \%$ of the students are not prepared to practice independently. Cohorts from Traditional curriculums (68\%) are more confident than Integrated curriculums $(53 \%)$. The results of this study were similar to study reported from India, in which only 64 percent of the students rated themselves as they are prepared to start an independent practice [13]. The percentage of students' preparedness to practice was less in this study compared to other similar study conducted in Hong Kong, where $72 \%$ of the students indicated their well-preparedness to practice [14].

Students from both the cohorts are well prepared when it comes to "Bread and butter" of dental practice such as dental practice management, diagnosis, simple extractions and restorations. The skills that involve recording patient case history, diagnostic accuracy, treating, and prevention of oral diseases have been labeled in the literature as the bread and butter items in general dentistry $[15,16]$. Such basic procedures in clinical practice, executed almost every day by students, and it is reassuring to learn that both cohorts were proficient in doing these procedures. This aspect is comparable with students' self-perceived preparedness for a dental practice in other countries like Australia [17], and West Indies [18].

Overall scores of Traditional curriculum students $(176 \pm 4.8)$ were more compared to Integrated curriculum students $(161 \pm 3.2)$ and across the ten items (domains) of dental clinical practice, students' selfrated competencies were least for Knowledge in applying evidence-based dentistry followed by performing surgical procedure for clinical crown lengthening, Endodontic procedure for posterior tooth, Orthodontic treatment planning and ability to manage medically compromised patients. Almost similar results were reported from various studies globally. The three most competent self-perceived competencies reported from a study done in Canada are recording the patient's case history, recognizing chief complaint of the patient, and diagnosis [7]. Students in Trinidad and Tobago reported that they are more confident in recording an adequate medical history, diagnosing and treating dental caries, performing an oral examination, and providing appropriate dental health education to the patients [18]. Students in Australia expressed that they were very competent to record and interpret patient case history, Endodontic treatment and rehabilitation for single root teeth, and perform deep scaling and root planning [17].

Among both cohorts, Integrated curriculum students had more self-perceived skill scores in Orthodontics, Conservative/Operative Dentistry \& Endodontics, Dental Public Health and Drug \& Emergency Management. In orthodontics, both the cohorts scored themselves as less competent. In both types of undergraduate curriculums, orthodontics was covered in fourth and fifth academic years and less emphasis on clinical training in Orthodontics compared to other subjects [19]. Students learn mainly basic wire bending exercises and dental model analysis for space-regaining procedures and they don't directly deal with orthodontic patients as orthodontics practice has been projected as a development that has occurred in postgraduate training.

There are few limitations in our study. Firstly, the self-reported nature of the information, which may be different from actual clinical competence of the trainees, but this study mainly addressed the appropriateness of dental curriculum and opinions of the students as a stakeholders play an important role in finalizing the 
curriculum [12]. Secondly, the time gap since completion of graduation and clinical experience may affect the interpretation and response to the surveys, to avoid such gap only graduating dental students who are to be graduated in 2020 are included in this study and current students will have fresh memory about their dental program. However, having certain limitations, the findings of this study will contribute for assessing the appropriateness of both dental curriculums in students' perspectives and helps in specifically for designing Undergraduate (BDS) dental curriculum. Hence, these gaps will need to be addressed in designing future curriculum of the dental graduation course.

\section{Conclusion}

This study found that, as a whole, students have a poor perception about their preparedness to practice. The Ministry of Education in Saudi Arabia should take necessary action about concerns perceived by the students to bridge the gap in making the dental students to be competent practitioners.

\section{Authors' Contributions}

SV (D) 0000-0003-0613-5179 Conceptualization, Methodology, Investigation, Writing - Original Draft Preparation and Writing - Review and Editing.

The author declares that he contributed to critical review of intellectual content and approval of the final version to be published.

\section{Financial Support}

None.

\section{Conflict of Interest}

The authors declare no conflicts of interest.

\section{References}

[1] General Authority for Statistics. Kingdom of Saudi Arabia. [Available from: https://www.stats.gov.sa/sites/default/files/population_by_age_groups_and_gender_en.pdf. [Accessed on November 11, 2019.]

[2] AlBaker AA, Al-Ruthia YSH, AlShehri M, Alshuwairikh S. The characteristics and distribution of dentist workforce in Saudi Arabia: a descriptive cross-sectional study. Saudi Pharm J 2017; 25(8):1208-16. https://doi.org/10.1016/j.jsps.2017.09.005

[3] Al-Shalan TA. Dental education in Saudi Arabia: areas of attention. Saudi Dent J 2018; 30(4):271-2. https://doi.org/10.1016/j.sdentj.2018.06.005

[4] Pogrel MA. Dental education in the US. Br Dent J 2014; 217(10):557-8. https://doi.org/10.1038/sj.bdj.2014.999

[5] Mahal AS, Shah N. Implications of the growth of dental education in India. J Dent Educ 2006; 70(8):884-91.

[6] Al-Madi EM, Celur SL, Nasim M. Effectiveness of PBL methodology in a hybrid dentistry program to enhance students' knowledge and confidence. (a pilot study). BMC Med Educ 2018; 18(1):270. https://doi.org/10.1186/s12909-018-1392-y

[7] Al-Madi EM, AlShiddi M, Al-Saleh S, AbdelLatif H. Developing a dental curriculum for the 21 st century in a new dental school in Saudi Arabia. J Dent Educ 2018; 82(6):591-601. https://doi.org/10.21815/JDE.018.066

[8] Yiu CK, McGrath C, Bridges S, Corbet EF, Botelho M, Dyson J, et al. Graduates' perceived preparedness for dental practice from PBL and traditional curricula. J Dent Educ 2011; 75(9):1270-9.

[9] Plasschaert A, Boyd M, Andrieu S, Basker R, Beltran RJ, Blasi G, et al. Development of professional competences. Eur J Dent Educ 2002; 6(Suppl 3):33-44. https://doi.org/10.1034/j.1600-0579.6.s3.5.x

[10] Al-Damegh SA, Baig LA. Comparison of an integrated problem-based learning curriculum with the traditional discipline-based curriculum in KSA. J Coll Physicians Surg Pak 2005; 15(10):605-8.

[11] American Association of Dental Schools. Competencies for the new dentist (as approved by the 1997 House of Delegates). J Dent Educ 2007; 71(7):926-8. 
[12] Yusof ZY, Jaafar N, Jallaludin RLR, Abu-Hassan MI, Razak IA. Malaysian dental students' competence in holistic care: what do students and employers think? J Dent Educ 2010; 74(12):1380-7.

[13] Shetty VB, Shirahatti RV, Pawar P. Students' perceptions of their education on graduation from a dental school in India. J Dent Educ 2012; 76(1):1520-6.

[14] Yiu CK, McGrath C, Bridges S, Corbet EF, Botelho MG, Dyson JE, et al. Self-perceived preparedness for dental practice amongst students of The University of Hong Kong's integrated PBL dental curriculum. Eur J Dent Educ 2012; 16(1):e96-105. https://doi.org/10.1111/j.1600-0579.2011.00681.x

[15] Greenwood LF, Lewis DW, Burgess RC. How competent do our graduates feel? J Dent Educ 1998; 62(4):307-13.

[16] DeCastro JE, Matheson PB, Panagakos FS, Stewart DC, Feldman CA. Alumni perspectives on community-based and traditional curricula. J Dent Educ 2003; 67(4):418-26.

[17] Arena G, Kruger E, Holley D, Millar S, Tennant M. Western Australian dental graduates' perception of preparedness to practice: a five-year follow-up. J Dent Educ 2007; 71(9):1217-22.

[18] Rafeek RN, Marchan SM, Naidu RS, Carrotte PV. Perceived competency at graduation among dental alumni of the University of the West Indies. J Dent Educ 2004; 68(1):81-8.

[19] Yusof ZY, Jaafar N, Jallaludin RL, Abu-Hassan MI, Razak IA. Malaysian dental graduates' competence in holistic care: what do graduates and employers think? J Dent Educ 2010; 74(12):1380-7. 